\title{
Índice de normalidade e confiabilidade do pick up test de Moberg (modificado por Dellon) para o nervo mediano ${ }^{1}$
}

\author{
Camila Caroline Loss ${ }^{\mathrm{a}}$, Regyane Costa ${ }^{\mathrm{b}}$, Therezinha Rosane Chamlian ${ }^{\mathrm{c}}$ \\ ${ }^{a}$ Fisioterapeuta, Especialista em Terapia da Mão, Escola Paulista de Medicina - EPM, \\ Universidade Federal de São Paulo - UNIFESP, São Paulo, SP, Brasil \\ ${ }^{\text {b} T e r a p e u t a ~ O c u p a c i o n a l, ~ E s p e c i a l i s t a ~ e m ~ T e r a p i a ~ d a ~ M a ̃ o, ~ F a c u l d a d e ~ d e ~ M e d i c i n a ~ d a ~ U n i v e r s i d a d e ~}$ \\ de São Paulo - FMUSP, Coordenadora e Supervisora da Pós-Graduação lato sensu \\ em Terapia da Mão e Membro Superior, Escola Paulista de Medicina - EPM, \\ Universidade Federal de São Paulo - UNIFESP, São Paulo, SP, Brasil \\ 'Fisiatra, Doutora, Professora afiliada do Departamento de Ortopedia e Traumatologia, \\ Escola Paulista de Medicina - EPM, Universidade Federal de São Paulo - UNIFESP, \\ Diretora Técnica, Centro de Reabilitação Lar Escola São Francisco, São Paulo, SP, Brasil
}

\begin{abstract}
Resumo: O pick up test modificado por Dellon avalia a capacidade e a habilidade da mão em identificar pequenos objetos com e sem o uso da visão. Objetivos: Elaborar uma padronização para a sua utilização e estabelecer o índice de normalidade para a população adulta; identificar se existem diferenças entre o tempo de execução dos subtestes para as variáveis: gênero, mão direita e esquerda, mão dominante e não dominante, nível de escolaridade, além de avaliar a confiabilidade interexaminadores. Materiais e Métodos: Foi realizado um estudo transversal com 100 voluntários da UNIFESP - SP, sem lesão nos membros superiores. Um total de 10 objetos conhecidos pelo participante foi disposto à sua frente, sendo ele instruído a colocar um por vez dentro de uma caixa o mais rápido possível. Este teste foi realizado com os olhos abertos e fechados. Para verificar a confiabilidade interexaminadores, os participantes foram avaliados por duas pesquisadoras. Resultados: Não houve diferenças estatisticamente significantes entre as avaliadoras e os gêneros. Foram encontradas correlações estatisticamente significantes entre a mão direita e a esquerda e para o nível de escolaridade. Conclusão: A média geral de tempo em segundos para os olhos abertos com a mão direita foi de 18,23 s e com a mão esquerda, 19,96 s; para os olhos fechados, com a mão direita, foi de 37,54 s e com a mão esquerda, 39,42 s. A média de tempo dos participantes com escolaridade de nível fundamental apresentou-se maior em relação aos demais níveis. O pick up test de Moberg modificado por Dellon é um teste reprodutível.
\end{abstract}

Palavras-chave: Pick up Test, Nervo Mediano, Destreza Motora, Terapia Ocupacional.

\section{Normality and reliability of Moberg pick up test (modified by Dellon) for median nerve}

\begin{abstract}
Moberg Pick up Test modified by Dellon evaluates the person's hand ability to identify small objects with and without the use of eyesight. Purpose: Developing a standard for its use and establishing the normalcy index for the adult population in order to identify whether there are differences between the runtime of the subtests for variables gender, right and left dominant and non-dominant hand, educational level; and to evaluate the interrater's reliability. Methods: A cross-sectional research was carried out with 100 volunteers from the Federal University of Sao Paulo (UNIFESP-SP) that presented no lesion in the upper limbs. A total of 10 objects known by the participants were placed in front of them and they were instructed to place one at a time inside a
\end{abstract}

Autor para correspondência: Camila Caroline Loss, Rua Professor Cristiano Fischer, 818/701, Petrópolis, CEP 91410-000, Porto Alegre, RS, Brasil, email: camilaloss@gmail.com

Recebido: 30/11/2010; $1^{\text {a }}$ Revisão: 11/7/2011; $2^{\text {a }}$ Revisão: 28/11/2011; Aceite final: 8/2/2012. 
box as quickly as possible. This test was performed with open and closed eyes. In order to check the interrater's reliability, the participants were evaluated by two researchers. Results: There were no statistically significant differences between raters and genders. There were statistically significant correlations between left and right hands and the educational level. Conclusion: The overall average runtime in seconds to open eyes with the right hand was $18.23 \mathrm{~s}$ and with the left hand, $19.96 \mathrm{~s}$; to closed eyes with the right hand, $37.54 \mathrm{~s}$ and with the left hand, $39.42 \mathrm{~s}$. The mean time for primary level was greater than for the other levels. Moberg Pick up Test modified by Dellon is a reproducible test.

Keywords: Pick up Test, Median Nerve, Motor Skills, Occupational Therapy.

\section{Introdução}

Moberg (1958 apud SAMARÃO, 1984) introduziu o termo gnosia tátil, que se refere especificamente à percepção da sensibilidade e função presente na ponta dos dedos. Essa é uma função que permite a percepção e localização do toque, a discriminação e o reconhecimento das qualidades e identificaçóes de objetos sem usar a visão. $\mathrm{O}$ dedo indicador, por sua vez, é o mais rico em terminaçóes sensitivas e, por isso, é fundamental na exploração tátil.

Os nervos periféricos são prolongamentos do sistema nervoso central, responsáveis pela integração das atividades sensitivas e motoras da mão. O nervo mediano faz referência à sensibilidade, tornando-se o mais importante, pois inerva as superfícies palmares do polegar, indicador, médio e metade do anular. É considerado um nervo informador, pois essa é a principal área discriminativa da mão que permite o reconhecimento de objetos (PARDINI; FREITAS, 2008).

Pacientes com lesão do nervo mediano apresentam déficits motores e sensitivos. Como consequências, desenvolvem o quadro clínico característico de paralisia e hipotrofia da musculatura tenar, musculaturas intrínseca e extrínseca da mão, resultando em dificuldades para manusear e identificar objetos, o que gera um grande prejuízo funcional (PARDINI; FREITAS, 2008).

A avaliação das sensaçôes somáticas é pesquisada quando há hipótese de distúrbios, compressões e doenças relacionadas ao sistema nervoso (FERRIGNO, 2007). Os instrumentos utilizados para investigaçáo e documentação dos resultados clínicos após uma lesão nervosa periférica devem ser precisos. Os testes devem auxiliar na determinação do grau de reinervação e denervação, colaborar com o diagnóstico, além de avaliar a condição sensorial e motora (HEROLD, 2005; SBTM, 2005). De acordo com as recomendaçóes para a avaliação do membro superior, preconizadas pela Sociedade Brasileira de Terapeutas da Máo (SBTM), os instrumentos ainda necessitam de mais estudos no que se refere aos índices de normalidade, validade e confiabilidade (SBTM, 2005).

Segundo Atchison (1987), os testes padronizados incluem instruções específicas para a sua administração e pontuação e contêm evidências estatísticas de validade e confiabilidade. $\mathrm{O}$ autor relata que cada vez mais os terapeutas ocupacionais têm sido encorajados a utilizar estes testes, a fim de melhorar a formalização da avaliação de Terapia Ocupacional com base em dados quantitativos. Desta forma, os resultados das avaliaçóes dos pacientes podem ser relatados de maneira consistente, objetiva e confiável, além disso, o uso de avaliaçóes padronizadas aumenta a credibilidade profissional (ATCHISON, 1987; WATSON, 1983).

Segundo Dellon (1997), Moberg pode ter sido um dos primeiros a enfatizar que um teste quantitativo deve ser válido e que um teste de sensibilidade também deve fornecer dados a respeito da função motora. "Confiabilidade", segundo a SBTM (2005), significa que diferentes examinadores, ao utilizarem o mesmo teste para avaliar a mesma área, com o mesmo assunto, cada um terá um resultado próximo da medida feita pelo outro examinador. Moberg em 1958 desenvolveu o pick up test, que avalia a capacidade e a habilidade da mão em identificar pequenos objetos com e sem o uso da visão. Este teste combina características essenciais para o reconhecimento de doze objetos, entre eles, parafuso, chave, clipes de papel, bolinha de gude, porca sextavada, moeda, etc. (HEROLD, 2003, 2005; SBTM, 2005; CAROL; DEREK; CHOW,1999). Cada indivíduo teria aproximadamente dois segundos para identificar cada um deles (DELLON, 1983). O teste depende fortemente do controle motor do polegar, indicador e dedo médio; é, portanto, útil para avaliar o nervo mediano (HEROLD, 2005). Os resultados do pick up test têm um valor funcional, porque, além de avaliar a estereognosia, refletem o desempenho 
manual (CAROL; DEREK; CHOW,1999). Dellon (1981 apud HEROLD, 2005), por acreditar que os pacientes reconheceriam os objetos do pick up test de Moberg pela temperatura e pela textura, desenvolveu uma versão modificada deste, para a qual preconizou apenas objetos de metal, exigindo assim sua identificação correta.

Herold (2005), através de uma revisão sistemática que teve como objetivo identificar os testes válidos e confiáveis disponíveis para avaliaçáo da sensibilidade das mãos, encontrou como resultados que o pick up test de Moberg e o pick up test de Moberg modificado por Dellon ainda não possuem estudos relacionados à confiabilidade (HEROLD, 2005).

Por meio de um protocolo padronizado, Carol, Derek e Chow, 1999, realizaram um estudo com objetivo de estabelecer os índices normativos para o pick up test de Moberg e avaliar a confiabilidade interexaminadores. Os resultados demonstraram que houve diferenças no tempo de execução do teste entre as variáveis gênero, mão dominante e náo dominante (CAROL; DEREK; CHOW, 1999).

$\mathrm{Na}$ literatura nacional não foram localizadas publicaçóes referentes aos índices de normalidade do pick up test de Moberg e do pick up test modificado por Dellon. A interpretaçáo dos resultados é comparada com a do lado contralateral sadio, o que pode ser inviabilizado, uma vez que o lado oposto pode apresentar acometimentos. Desta forma, uma preocupação das pesquisadoras foi estabelecer índices de normalidade para interpretar o que seria anormal, ou seja, dados que sugerissem a presença de déficits funcional e sensorial e a necessidade de intervenção terapêutica.

Os objetivos deste estudo foram:

- elaborar uma padronização para utilização do pick up test de Moberg modificado por Dellon;

- estabelecer o índice de normalidade;

- identificar se existem diferenças entre o tempo de execuçáo dos subtestes para as variáveis gênero, mão direita e esquerda, mão dominante e não dominante e nível escolar;

- avaliar a confiabilidade interexaminadores.

\section{Materiais e métodos}

Realizou-se um estudo transversal com 100 voluntários adultos (18-59 anos), alfabetizados (ensinos fundamental, médio ou superior), de ambos os sexos, sem alteraçóes do sistema musculoesquelético nos membros superiores e sem alterações cognitivas de qualquer etiologia. Estes participantes foram acompanhantes de pacientes, profissionais da área da saúde e funcionários do Lar Escola São Francisco e do Hospital São Paulo.

O estudo foi aprovado pelo comitê de ética $(0832 / 09)$ e conduzido no serviço de Terapia da Máo e Membro Superior, do setor de Terapia Ocupacional do Lar Escola São Francisco e no ambulatório de Cirurgia da Mão e Membro Superior do departamento de Ortopedia e Traumatologia da Universidade Federal de São Paulo (UNIFESP/ EPM) (LOSS et al. 2009).

Os voluntários foram convidados aleatoriamente a participarem da pesquisa. Depois da assinatura do Termo de Consentimento Livre e Esclarecido em local que assegurasse privacidade, foram coletados os dados de caracterização dos voluntários e aplicado o pick up test de Moberg modificado por Dellon. O teste é composto por uma caixa medindo $30 \times 60 \mathrm{~cm}$ (Figura 1), um prego, um parafuso grande, um parafuso pequeno, um gancho, uma porca grande, uma porca pequena, uma arruela, uma chave, uma moeda e um alfinete (Figura 2).

Para testar sua confiabilidade, os participantes foram avaliados por duas pesquisadoras distintas, ambas terapeutas da mão, com um intervalo de tempo de dez minutos entre cada avaliação. Os cinquenta primeiros participantes foram avaliados pela terapeuta 1 . Com os demais participantes, a ordem das avaliadoras foi invertida, sendo a primeira avaliação realizada pela terapeuta 2 . Os

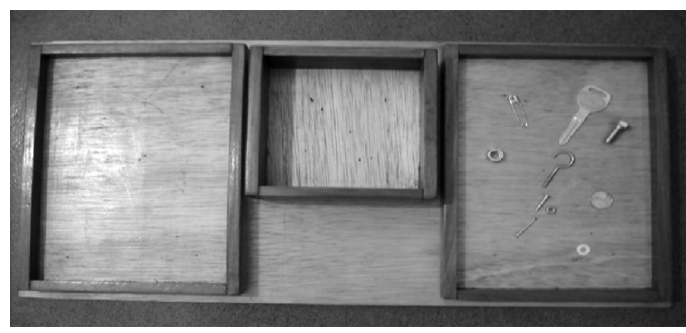

Figura 1. caixa do pick up test.

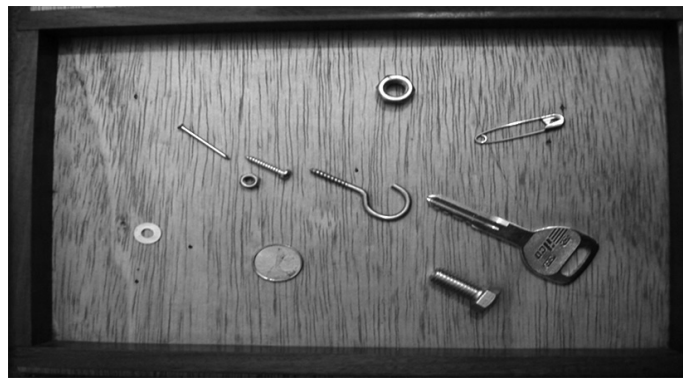

Figura 2. objetos do pick up test. 
participantes eram posicionados sentados, com o tronco apoiado no encosto da cadeira, quadril e joelhos fletidos a $90^{\circ}$, pés apoiados no chão e cotovelos fletidos a $90^{\circ}$ apoiados sobre mesa. Antes da aplicação do teste, foram apresentados a eles todos os objetos a serem discriminados, bem como suas respectivas denominaçóes. Foi solicitado aos participantes que colocassem em ambas as mãos luvas de látex no $4^{\circ}$ e $5^{\circ}$ dedos, com a finalidade de serem utilizados somente o polegar, indicador e médio no ato da discriminação dos objetos. A seguir, foi solicitado a eles que pegassem um objeto de cada vez, primeiramente com a mão não dominante, $o$ nomeassem e o colocassem numa caixa localizada a sua frente o mais rápido possível, até completar o número de dez objetos. Primeiramente, o teste foi realizado com os olhos abertos com a mão direita e depois com a esquerda. Posteriormente o mesmo teste foi repetido com os olhos vendados. Ao final destes, o tempo foi cronometrado e os dados foram anotados na ficha de avaliação.

Os resultados foram submetidos à análise estatística descritiva, aplicação do teste de Wilcoxon, teste de Mann-Whitney, teste de Kruskl-Wallis, e teste de Igualdade de Duas Proporçôes. Para todos os testes estatísticos, o nível de significância adotado foi de $0,05 \%$ com intervalos de confiança de $95 \%$.

Quanto a caracterização da amostra estudada observa-se por meio da Tabela 1, que 51\% dos voluntários foram do sexo feminino. A média de idade foi de 36,2 anos, com predominância do membro dominante direito (96\%). Em relação ao nível de escolaridade $51 \%$ possuiam nível superior.

\section{Resultados}

$\mathrm{Na}$ Tabela 2, é apresentada a média de tempo em segundos em que os voluntários executaram o teste, bem como as correlaçôes entre as variáveis: mão direita e mão esquerda, com olhos abertos e fechados. O tempo médio para os olhos abertos com a mão direita foi de $18,23 \mathrm{~s}$, e com a mão esquerda, 19,96 s; para olhos fechados com a mão direita foi de $37,54 \mathrm{~s}$, e com a mão esquerda, 39,42 s. O escore médio do Coeficiente de Variação (CV) foi de 33\%, demonstrando que os dados são homogêneos.

A Tabela 3 apresenta a comparação da média de tempo entre os gêneros. As mulheres realizaram os subtestes em um tempo menor; entretanto, essa diferença não foi considerada estatisticamente significante.

Em relação ao nível de escolaridade, a Tabela 4 apresenta uma diferença estatisticamente significante,
Tabela 1. Caracterização da amostra.

\begin{tabular}{ll}
\hline Sexo & $51 \%$ feminino \\
& $49 \%$ masculino \\
\hline Idade média & 36,2 anos \\
\hline Dominância & $96 \%$ destros \\
\hline & $51 \% \mathrm{ES}$ \\
Escolaridade & $26 \% \mathrm{EM}$ \\
& $21 \% \mathrm{EF}$ \\
& $2 \% \mathrm{ET}$ \\
\hline
\end{tabular}

$\mathrm{ES}=$ Ensino superior, $\mathrm{EM}=$ Ensino médio, $\mathrm{EF}=$ Ensino Fundamental, ET = Ensino técnico.

pois os participantes com ensino fundamental realizaram os subtestes em maior tempo, quando comparados aos demais.

Em relação à confiabilidade interexaminadores, a Tabela 5 demonstra que não houve diferença estatisticamente significante entre as avaliadoras, já que $\mathrm{p}<0,05$.

As Tabelas 6 e 7 apresentam, respectivamente, os resultados encontrados após a mudança na ordem das avaliadoras. Constatou-se que houve correlaçóes estatisticamente significantes, pois, sempre que avaliados pela segunda vez, os participantes executaram os testes em menor tempo.

\section{Discussão}

A busca de dados quantitativos sobre a função da mão após a lesão traumática do nervo mediano nos motivou a estudar o pick up teste de Moberg modificado por Dellon. Este é considerado um teste funcional, pois avalia a capacidade e a habilidade da mão com e sem o uso da visão.

De acordo com Herold (2005), o pick up teste de Moberg ainda não possui estudos relacionados à confiabilidade (HEROLD, 2005). A SBTM (2005) por sua vez, relata que estes necessitam de mais estudos sobre a normalidade, a validade e a confiabilidade, fato que se confirma através da literatura, na qual encontrou-se apenas o artigo de Carol, Derek e Chow (1999) semelhante a este estudo.

A padronização de qualquer avaliação está diretamente relacionada com a reprodutibilidade, o que permite resultados mais confiáveis. Carol, Derek e Chow (1999), foram sucintos em relaçáo ao posicionamento dos participantes e optaram por iniciar o teste com a mão dominante. Sabe-se que a máo dominante geralmente é a mais acometida por diferentes lesóes, por isso, diferentemente do estudo de Carol, Derek e Chow (1999), nesta pesquisa optou-se por iniciar com a mão não dominante, já que ao iniciar o primeiro subteste, os participantes poderiam 
Tabela 2. Índice de normalidade.

\begin{tabular}{lcccc}
\hline \multirow{2}{*}{ Descritiva } & \multicolumn{2}{c}{ Olho aberto } & \multicolumn{2}{c}{ Olho fechado } \\
\cline { 2 - 5 } & Mão direita & Mão esquerda & Mão direita & Mão esquerda \\
\hline Média & 18,23 & 19,96 & 37,54 & 39,42 \\
Desvio padrão & 5,97 & 6,48 & 14,32 & 13,07 \\
CV $(\%)$ & 33 & 32 & 38 & 33 \\
\hline
\end{tabular}

$\mathrm{CV}=$ Coeficiente de variação.

Tabela 3. Compara gênero nos testes.

\begin{tabular}{lcccccccc}
\hline \multirow{2}{*}{ Sexo } & \multicolumn{2}{c}{$\begin{array}{c}\text { Olho aberto } \\
\text { mão direita }\end{array}$} & \multicolumn{2}{c}{$\begin{array}{c}\text { Olho aberto } \\
\text { mão esquerda }\end{array}$} & \multicolumn{2}{c}{$\begin{array}{c}\text { Olho fechado } \\
\text { mão direita }\end{array}$} & \multicolumn{2}{c}{$\begin{array}{c}\text { Olho fechado } \\
\text { mão esquerda }\end{array}$} \\
\cline { 2 - 10 } & Fem. & Masc. & Fem. & Masc. & Fem. & Masc. & Fem. & Masc. \\
\hline Média & 18,04 & 18,43 & 19,35 & 20,59 & 36,90 & 38,21 & 38,95 & 39,91 \\
Desvio padrão & 6,39 & 5,53 & 6,20 & 6,73 & 16,72 & 11,36 & 13,56 & 12,59 \\
p-valor & 0,208 & & 0,154 & & 0,063 & & 0,334 & \\
\hline
\end{tabular}

Tabela 4. Compara escolaridade nos testes.

\begin{tabular}{ccccc}
\hline & Escolaridade & Média & Desvio padrão & p-valor \\
\hline \multirow{3}{*}{ Olho aberto mão direita } & EF & 20,85 & 7,37 & \\
& EM & 18,02 & 4,14 & 0,001 \\
& ES & 17,41 & 5,94 & \\
\hline \multirow{3}{*}{ Olho aberto mão esquerda } & EF & 22,64 & 7,04 & \\
& EM & 20,14 & 5,90 & $<0,001$ \\
& ES & 18,95 & 6,29 & \\
Olho fechado mão direita & EF & 44,12 & 21,87 & $<0,001$ \\
& EM & 37,27 & 10,85 & \\
& ES & 35,41 & 11,10 & $<0,001$ \\
Olho fechado mão esquerda & EF & 45,50 & 15,23 & \\
& EM & 39,74 & 12,31 & \\
\hline
\end{tabular}

Tabela 5. Confiabilidade interexaminadores.

\begin{tabular}{lcccccccc}
\hline Avaliadores & \multicolumn{2}{c}{$\begin{array}{c}\text { Olho aberto } \\
\text { mão direita }\end{array}$} & \multicolumn{2}{c}{$\begin{array}{c}\text { Olho aberto } \\
\text { mão esquerda }\end{array}$} & \multicolumn{2}{c}{$\begin{array}{c}\text { Olho fechado } \\
\text { mão direita }\end{array}$} & \multicolumn{2}{c}{$\begin{array}{c}\text { Olho fechado } \\
\text { mão esquerda }\end{array}$} \\
\cline { 2 - 10 } & Aval 1 & Aval 2 & Aval 1 & Aval 2 & Aval 1 & Aval 2 & Aval 1 & Aval 2 \\
\hline Média & 18,26 & 18,21 & 20,31 & 19,61 & 38,01 & 37,07 & 39,71 & 39,13 \\
Desvio padrão & 5,74 & 6,23 & 6,89 & 6,05 & 17,03 & 11,04 & 14,30 & 11,78 \\
p-valor & 0,929 & & 0,941 & & 0,954 & & 0,930 & \\
\hline
\end{tabular}

Tabela 6. Avaliação dos 50 primeiros participantes.

\begin{tabular}{lcccccccc}
\hline \multirow{2}{*}{$\begin{array}{c}\text { Momentos } \\
\text { (50 primeiros) }\end{array}$} & \multicolumn{2}{c}{$\begin{array}{c}\text { Olho aberto } \\
\text { mão direita }\end{array}$} & \multicolumn{2}{c}{$\begin{array}{c}\text { Olho aberto } \\
\text { mão esquerda }\end{array}$} & \multicolumn{2}{c}{$\begin{array}{c}\text { Olho fechado } \\
\text { mão direita }\end{array}$} & \multicolumn{2}{c}{$\begin{array}{c}\text { Olho fechado } \\
\text { mão esquerda }\end{array}$} \\
\cline { 2 - 10 } & Pré & Pós & Pré & Pós & Pré & Pós & Pré & Pós \\
\hline Média & 19,63 & 17,29 & 22,72 & 17,31 & 43,27 & 35,45 & 45,31 & 38,38 \\
Desvio padrão & 6,56 & 5,99 & 7,85 & 4,67 & 21,05 & 12,29 & 15,71 & 12,08 \\
p-valor & 0,001 & & $<0,001$ & & $<0,001$ & & $<0,001$ & \\
\hline
\end{tabular}

Pré $=$ Terapeuta 1, Pós = Terapeuta 2. 
Tabela 7. Avaliação dos 50 últimos participantes.

\begin{tabular}{|c|c|c|c|c|c|c|c|c|}
\hline \multirow[t]{2}{*}{$\begin{array}{l}\text { Momentos } \\
\text { (50 últimos) }\end{array}$} & \multicolumn{2}{|c|}{$\begin{array}{l}\text { Olho aberto } \\
\text { mão direita }\end{array}$} & \multicolumn{2}{|c|}{$\begin{array}{l}\text { Olho aberto } \\
\text { mão esquerda }\end{array}$} & \multicolumn{2}{|c|}{$\begin{array}{c}\text { Olho fechado } \\
\text { mão direita }\end{array}$} & \multicolumn{2}{|c|}{$\begin{array}{l}\text { Olho fechado } \\
\text { mão esquerda }\end{array}$} \\
\hline & Pré & Pós & Pré & Pós & Pré & Pós & Pré & Pós \\
\hline Média & 19,12 & 16,88 & 21,92 & 17,89 & 38,68 & 32,76 & 39,87 & 34,10 \\
\hline Desvio padrão & 6,39 & 4,43 & 6,42 & 4,73 & 9,48 & 9,32 & 11,54 & 10,11 \\
\hline p-valor & \multicolumn{2}{|c|}{$<0,001$} & \multicolumn{2}{|c|}{$<0,001$} & \multicolumn{2}{|c|}{$<0,001$} & \multicolumn{2}{|c|}{$<0,001$} \\
\hline
\end{tabular}

Pré $=$ Terapeuta 2, Pós = Terapeuta 1.

estar apreensivos, interferindo negativamente no tempo de discriminação dos objetos. Após a primeira experiência, cientes do método de avaliação, os participantes estariam mais tranqüilos ao realizar o teste, sem que houvesse interferência no tempo de discriminação dos objetos. É importante ressaltar que essa preocupação foi apenas para estabelecer índices normativos; ao reproduzir este estudo, os avaliadores poderão iniciar o teste com a máo lesada. Nesta pesquisa, houve preocupação com a utilização de 10 objetos de metal do teste original do pick up teste (modificado por Dellon), (um prego, um parafuso grande, um parafuso pequeno, um gancho, uma porca grande, uma porca pequena, uma arruela, uma chave, uma moeda e um alfinete).

Em relação ao gênero, observou-se que, embora os participantes do sexo feminino tenham executado os subtestes em tempo menor que os do sexo masculino, os resultados não foram considerados estatisticamente significantes, diferentemente de Carol, Derek e Chow (1999), que encontraram resultados estatisticamente significantes, no qual as mulheres realizaram grande parte dos subtestes em menor tempo.

Nas comparaçôes entre as variáveis mão direita, mão esquerda, mão dominante e mão não dominante, verificou-se que os participantes que possuíam a mão direita como dominante (96\%), o tempo de execução com os olhos abertos $(18,23 \mathrm{~s})$ e fechados (37,54 s) foi menor em todos os subtestes quando comparados com a mão esquerda, olhos abertos $(19,96 \mathrm{~s})$ e fechados (39,42 s). Os participantes com a máo esquerda como dominante $(4 \%)$, também executaram os subtestes em menor tempo quando comparados com a mão direita, inferindo que a execução com a mão dominante resulta em tempos menores. Apesar de Carol, Derek e Chow (1999) não terem realizado a comparação entre as variáveis mão direita e mão esquerda, também concluíram que o tempo de execução dos subtestes com a mão dominante com olhos abertos e fechados são menores em relação à mão náo dominante.

Carol, Derek e Chow (1999) não fizeram a correlação com a variável nível de escolaridade. Por acreditar que essa é uma variável que pode modificar os resultados do teste e para que os índices normativos da pesquisa ficassem mais equilibrados, foi feita essa correlação. Os resultados encontrados evidenciaram diferença estatisticamente significante entre o tempo de execução do teste dos participantes com ensino fundamental, que necessitam de maior tempo para execução em relação aos participantes com ensino médio e superior. A hipótese que pode justificar este resultado está relacionada à dificuldade que estes participantes manifestaram em memorizar o nome de alguns objetos.

No que se refere à avaliação da confiabilidade do teste, houve uma preocupação das pesquisadoras com a troca da ordem das avaliadoras, pois havia a hipótese de que os participantes, quando fossem avaliados pela segunda vez, levariam menos tempo para executar os subtestes. Assim, 50 participantes foram primeiramente avaliados pela pesquisadora 1 , e com os demais a ordem das avaliadoras foi invertida, ou seja, a pesquisadora 2 passou a ser a primeira avaliadora. Os resultados evidenciaram que independentemente da ordem das avaliadoras, os participantes quando avaliados pela segunda vez, de fato executavam os subtestes mais rapidamente. Em concordância com o estudo de Carol, Derek e Chow (1999) os resultados demonstraram que o pick up teste modificado por Dellon possui confiabilidade interexaminadores, o que o torna reprodutível.

\section{Conclusão}

Elaborou-se um protocolo de procedimentos para utilização do pick up test modificado por Dellon. A média geral de tempo em segundos que os participantes levaram para discriminar os 10 objetos com olhos abertos com a mão direita foi de $18,23 \mathrm{~s}$ e com a mão esquerda, $19,96 \mathrm{~s}$; olhos fechados, com a máo direita, $37,54 \mathrm{~s}$, e com a mão esquerda, 39,42 s. A média de tempo de execução dos participantes com nível fundamental apresentou-se maior em relação aos demais níveis. O pick up test de Moberg modificado por Dellon é reprodutível. 


\section{Referências}

ATCHISON, B. Selecting appropriate assessments, physical disabilities special interest section newsletter. American Journal of Occupational Therapy, v. 10, p. 2, 1987. CAROL, L.; DEREK, D.; CHOW, S. P. The Moberg pick up test. Results of testing with a standard protocol. Journal of Hand Therapy, v. 12, n. 4, p. 309-12, 1999. http://dx.doi.org/10.1016/S0894-1130(99)80069-6

DELLON, A. L. Evaluation of functional sensation in the hand. Journal of Hand Therapy, v. 8, n. 6, p. 865-70, 1983.

DELLON, A. L. Somatosensory testing e rehabilitation. Bethesda: American Occupational Therapy Association, 1997.

FERRIGNO, I. S. V. Terapia da Mão: Fundamentos para a prática clínica. São Paulo: Santos, 2007.

HEROLD, J. C. A study of the relative responsiveness of five sensibility tests for assessment of recovery after median nerve injury repair. Journal of Hand Therapy, v. $28 \mathrm{~B}$, n. 3 , p. $255-60,2003$.
HEROLD, J. C. Assesment of sensibility after nerve injury and repair: a systematic review of evidence for validity, reliability and responsiveness of tests. Journal of Hand Therapy, v. 30B, n. 3, p. 252-64, 2005.

LOSS, C. et al. Indice de normalidade e confiabilidade o pick up teste de Moberg (modificado por Dellon) para os dedos invervados pelo nervo mediano. 2009. $29 \mathrm{f}$. Monografia (Especialização em Terapia da Mão e Membro Superior)-Universidade Federal de São Paulo, São Paulo, 2009.

PARDINI, A.; FREITAS, A. Traumatismos da mão. Rio de Janeiro: Medbook, 2008.

SAMARÃO, B. Desenvolvimento psicomotor da mão. Rio de Janeiro: Enelivros, 1984.

SOCIEDADE BRASILEIRA DE TERAPIA DA MÁO E DO MEMBRO SUPERIOR - SBTM. Recomendações para avaliação do membro superior. Joinvile: SBTM, 2005.

WATSON, M. Analysis: Standardized testing objectives, physcial disabilities special interest section newsletter. American Journal of Occupational Therapy, v. 6, p. 4,1983 .

\section{Contribuição dos Autores}

Regyane Costa: concepçáo do manuscrito, pesquisa bibliográfica, redação do texto e revisão. Camila Loss: pesquisa bibliográfica, redação do texto e revisão. Os demais são revisores.

\section{Notas}

${ }^{1}$ Estudo desenvolvido e apresentado em forma de trabalho de conclusão de curso para aquisição de título de Especialização em Terapia da Mão e Membro Superior, na Universidade Federal de São Paulo (UNIFESP/EPM), desenvolvido no Departamento de Ortopedia e Traumatologia da UNIFESP. 